\title{
Me and My Robot Smiled at One Another: The Process of Socially Enacted Communicative Affordance in Human-Machine Communication
}

\author{
Carmina Rodríguez-Hidalgo ${ }^{1}$ \\ 1 School of Communications and Journalism, Universidad Adolfo Ibáñez, Viña del Mar, Chile
}

\begin{abstract}
The term affordance has been inconsistently applied both in robotics and communication. While the robotics perspective is mostly object-based, the communication science view is commonly user-based. In an attempt to bring the two perspectives together, this theoretical paper argues that social robots present new social communicative affordances emerging from a two-way relational process. I first explicate conceptual approaches of affordance in robotics and communication. Second, a model of enacted communicative affordance in the context of Human-Machine Communication (HMC) is presented. Third and last, I explain how a pivotal social robot characteristic_embodiment-plays a key role in the process of social communicative affordances in HMC, which may entail behavioral, emotional, and cognitive effects. The paper ends by presenting considerations for future affordance research in HMC.
\end{abstract}

Keywords: affordance, social robot, robotics, agency, interaction, human-machine communication

\section{Introduction}

Social robots have recently emerged as a new type of media with which we can communicate (Zhao, 2006). Social robots are quickly being adopted in our homes, they perform tasks in customer service, and can assist people with health issues or disabilities. Given their rapid spreading and their quickly improving capabilities, many regard the advent of social robots as part of the fourth industrial revolution (Cross et al., 2019). In their most sophisticated form, social robots are able to recognize, talk with, and personalize their interactions to communicate with humans (Guzman, 2018). Accordingly, in the field of human-machine 
communication (HMC) social robots are regarded as a new type of interaction partner (e.g., Edwards et al., 2019).

Despite the spreading and importance of social robots, our understanding of HMC awaits further study (Peter \& Kühne, 2018). Notably, albeit social robots increasingly acquire characteristics that, in principle, allow social or communicative uses, it is not clear whether people in fact perceive and act upon social robots as social and communicative entities. That is, we do not yet know whether social robots' characteristics do result in social communicative affordances, or action possibilities for communication (Gibson, 1979). For this reason, this article's first research question relates to what is the process of social communicative affordance formation when applied to human-machine communication (HMC). This, because we can hardly identify new social communicative affordances without understanding the process affordance formation first.

Further and beyond this, it is also not clear whether the idiosyncratic characteristics of social robots, such as their body and face, promote social and communicative uses which are comparable to the types of uses that emerge in interpersonal interactions or during media exposure. Social robots may have a social or communicative function for people, but they may fulfill this function in a different way than what is known between humans faceto-face or also when humans interact with each other through established communication technologies, such as Social Networking Sites (SNSs). Thus, it is conceivable that new forms of social interactions emerge in HMC. Wondering about how these salient social robot characteristics shape the interaction and effects from it brings this study's second research question, that is, what is the role of a social robot's embodiment in the process of social communicative affordance formation.

In the context of these pressing questions, I argue that the gap between social robots characteristics and people's social and communicative actions can be bridged by integrating a refined conceptualization of affordances into HMC. Integrating these approaches is necessary, as the term affordance has been inconsistently defined in the past. For instance, in robotics affordances have been mostly conceptualized in terms of the capabilities of robots to physically interact in their environment, such as recognizing and lifting objects (Horton et al., 2012; Paauwe et al., 2015). In contrast, communication science views affordances mostly in a human-centered way, focusing on what users can obtain from using technologies such as SNSs (boyd, 2010). While I readily acknowledge these important contributions, in our view, in the context of HMC, extant conceptualizations of affordances are rooted in a view of technology as a tool or medium of communication which does not sufficiently reflect the new reality that social robots are social communicators (Zhao, 2006). To integrate these approaches, the present article first develops a conceptualization of affordances which can be reasonably applied to HMC. Second, based on this conceptualization, I explain how physical embodiment lays in our view at the heart of enacted social communicative affordances between humans and social robots, particularly because of the sequential exchange of enhanced nonverbal communicative cues, such as haptic and audiovisual signals (e.g., voice intonation, facial expressions, physical touch). The study is structured as follows. I first explicate the conceptual underpinnings of the term affordance in both robotics and communication. Second, I examine main definitions of affordance and point to some inconsistencies in the conceptualization of the term. Third, and to shed light into the process of communicative affordance formation, I present a series of steps in a model of 
enacted affordance, which in our view may help better illustrate our point that affordances are enacted, rather than only perceived, in the particular context of HMC. Fourth, I present embodiment as a crucial element in this enacted affordance process. Fifth, and to clarify, I exemplify how embodiment is crucial to allow enacted social affordances.

\section{Grasping the Affordance Concept}

Originally stemming from ecological psychology, the concept of affordance was first mentioned by Gibson:

an affordance is what it offers the animal, what it provides or furnishes, for good or ill. The verb to afford is found in the dictionary, the noun affordance is not. I have made it up. I mean by it something that refers to both the environment and the animal in a way that no existing term does. It implies the complementarity of the animal and the environment. (Gibson, 1986, p. 127)

According to Gibson, affordances can be regarded as a particular characteristic of the environment which automatically triggers (and allows) an animal to carry out an action. For instance, a goat facing a hill would automatically perceive it as "climbable" due to its uphill and inclined morphology and would proceed to climb it. Here, the hill's morphology and shape are key to the action that is to be carried out. By extension, in the Gibsonian sense, the environment's shape and morphology both enable and determine actions. However, scholars quickly pointed out that not only the environments' characteristics, but also the capabilities of the animal influence which actions are performed. For instance, a goat is particularly skilled at climbing hills, whereas other animals, such as cows or butterflies, aren't proficient climbers, and would thus interact with the hill in a way which fits their specific abilities. In short, we can thus note that affordances are to be understood as the relation between the characteristics of a living entity and its environment.

\section{Affordances Applied to Technology}

Norman (1999) discussed affordances in terms of the action possibilities that are perceived by actors. His focus on human actors involved a move away from fully automatic responses and a stronger emphasis on interpretative and social processes and their influence on perceived action possibilities. For instance, when humans encounter a new tool, using it is likely to be the result of a cognitive evaluation of how it might be used and/or how other people use it, instead of a hard-wired automatic process. In line with this shifted focus, a newer definition of affordances appeared: "we view the affordances of an artifact as the possibilities (for both: thinking and doing) that are signified by users during their interaction with the artifact" (Vyas et al., 2006, p. 92). It is noteworthy that this newer view suggests that affordances are linked to social and subjective processes. Indeed, the socialconstructionist view of affordances puts the emphasis on users and how they construct (signify) the meaning about the artifact, which is influenced by social and cultural factors. As we discuss below, this social-constructionist view is at odds with Gibson's original conceptualization of affordances. However, it is exactly these differences between the Gibsonian 
and the social-constructionist view which are important to develop a comprehensive conceptualization of affordances in HMC.

\section{The Relationship Between Affordance and Perception}

As we have explained, in the Gibsonian conceptualization of affordance there exists an almost automatic relationship between perception and action. This notion of "direct perception," "sense of immediacy," or "automaticity" which is crucial in the Gibsonian framework, was critiqued by subsequent scholarship (see Stoffregen, 2003). For instance, cognitive scientists argued that perception of an object and its action possibilities could by no means be an automatic process, as perception is influenced by other factors, such as the optical abilities of subjects, or the memory of past experiences with that object (Fodor \& Pylyshyn, 1981; Horton et al., 2012).

This debate on the automaticity of affordances led other scholars to develop more refined definitions. Relevantly, Norman (1999) applied affordances to technological environments. He highlighted that affordances are first perceived, then enacted. For instance, when seeing a "clickable" button on a Web interface, Norman would reject to call the button itself strictly an affordance: "those displays are not affordances; they are visual feedback that advertise the affordances: they are the perceived affordances" (Norman, 2008). Therefore, if the person perceives a button as "clickable" and clicks on it and obtains a result (such as accessing another Web page), then the affordance manifests. This affordance could be, for instance, greater accessibility to more information online.

In addition, Norman (1999) posed that perceived affordances (e.g., the perceived possibilities for action) influence how people behave toward objects, especially technological ones. For instance, in the classic example of a chair, people may sit on it, lean on it, or even throw it to others depending on how people appraise its physical and social affordances (Pols, 2012). This conceptualization of perception as apart from action has found support in recent communication scholarship, which has considered "perceived affordances" (e.g., Fox \& Holt, 2018) to be more precise. Further, dealing with the issue of affordance and perception, Nagy \& Neff (2015) brought the concept of "imagined affordance" to highlight the importance of imagination in the affordance formation process, as "expectations for technology that are not fully realized in conscious, rational knowledge" (p. 1). These authors implied that affordances are not only perceived, they are in a large part imagined by users. This user-centered view has been common in communication approaches to affordances, as we will see below.

\section{The Concept of Affordances in Communication}

The conception of technology acting as a communication channel between two or more people played a role in the latest conceptualizations of affordances, which viewed them as emerging from the use of technology as a medium of communication. For instance, the field of human-computer interaction (HCI) identified key perceived affordances such as interactivity, which could result from either interacting with the computer or technology 
(e.g., clicking on a website link and gain access to new content), or emerging from communication between two humans mediated by a computer (e.g., two humans interacting with each other through e-mail). Even more recent conceptualizations have focused on the role of technology as enabling humans to connect and spread personal content in newer ways, for instance through Social Networking Sites (SNSs). From there, the field centered on how these social network platforms empower users, allowing greater connectivity and the diffusion of their own user-generated content. For instance, the notion of the scalability affordance (boyd, 2010) is that the visibility of users' content published in SNSs can easily escalate if other users reshare and spread content through various social networks. Other proposed affordances included persistence (online expressions are automatically recorded and archived) and searchability (online content can easily be accessed through search, boyd, 2010, p. 7).

In my view, a key recent communications approach on affordances proposed by Evans and colleagues (2016) emphasizes a relational view between people's actions and technology. In their definition of affordance, Evans et al. build on the relational view proposed by Faraj \& Azad (2012), whom stated that:

an affordance is a multifaceted relational structure, not just a single attribute or property or functionality of technology artifact or the actor. That is, affordance is often realized via the enactment of several mutuality relations between the technology, the artifact and the actor. (p. 254).

In my view, this definition emphasizes that affordances emerge enacted as a result of different relationships, which this article posits are a series of interrelated steps in the communicative process.

Consequently, Evans and colleagues (2016) focused on this relational stance and provided a conceptual definition of affordance as: “the multifaceted relational structure' (Faraj \& Azad, 2012, p. 254) between an object/technology and the user that enables or constraints potential behavioral outcomes in a particular context." From these definitions, it can be inferred that communication scholars mostly adhere to a conceptualization of affordances as a "multifaceted structure" and emphasize its relational character between the object, the features of the object, and the user. Moreover, Evans and colleagues proposed three criteria to assess whether a particular action would qualify as an affordance: (a) the affordance is neither the object nor the feature of the object; (b) the proposed affordance is not an outcome; and (c) the proposed affordance has variability. For (a) the authors explain that a "feature" represents a tool or attribute (p. 39) "that enables activity by part of the user" (Smock et al., 2011, p. 2323). For instance, the authors pose that a built-in camera on a phone is a feature, a tool which can be activated by the user, while the fact that one can capture photos or video, brings about the affordance of recordability. For (b), the outcomes, they argue that for example, easily finding a picture of someone we try to contact online, searching online for it for example, is an outcome of the affordance of accessibility (i.e., online content can easily be accessed, boyd, 2010). For (c) variability, Evans and colleagues propose that the affordance of visibility may vary (i.e., a photo may become more or less visible online). 


\section{Reassessing Communicative Affordances}

While I see much value in Evans et al. (2016) affordance definition and assessment criteria, such an "elastic" or interrelated conceptualization of affordances, at least when applied to HMC, may not allow for an explanation of all the constitutive elements of the perceived affordance process. Moreover, I believe that this valuable cornerstone definition provided by Evans and colleagues could be specified in three main ways. First, as I have shown in the previous sections, this definition could include user perception, as it is how interaction partners perceive the technology and the possibilities for action it allows, that which brings affordances. Here, I take distance from this "direct link" theorized by Gibson between perception and action, and are lenient to more current approaches, which signal that any effort to measure affordances should focus on perceived affordances. Second, this definition does not consider the issue of user agency. Agency is the feeling of oneself being the initiator of an action, the sense of self obtained through the perceived control over the social world (Brandi et al., 2019). Following these authors, I argue that any communicative situation between social actors includes agency, as the source A (for instance the human), should be willing to initiate a series of social actions (e.g., eye contact, making a question), and then receive feedback from source $B$ (the social robot) to that action (e.g., returning eye contact, answering the question). Following from this, I posit that it is not enough to be aware of the potential uses of technology, users need to want to use a technology as part of their personal agency with the objective of fulfilling a personally relevant goal.

Third and last, Evans et al.s (2016) definition does not really specify what outcomes are. Strictly speaking, everything can be an outcome, including affordances. For instance, persistence, argued by some scholars to be an affordance, can be an outcome from the action of image data capture. Or if a user willingly decides to never erase a social media post, content persistence is then also an outcome of that users' decision, apart from the particular features of the technology itself (e.g., servers with enough storage capacity to store personal content online). In this respect, I would like to specify that this paper defines outcomes in the context of HMC as the behavioral, attitudinal, and cognitive effects emerging from interactions with social robots. As we have seen, the concept of affordance has faced various definitions and approaches, which are not usually consistent. One example of a different approach is that of robotics, which I proceed to briefly discuss hereunder.

\section{The Concept of Affordance in Robotics}

The traditional application of affordances in robotics has focused heavily on how the robot moves and physically deals with objects and elements in the environment. For instance, how does the robot successfully enact movement (walk, jump, run, e.g., Kuindersma et al., 2016), how it can distinguish different elements, such as objects, people, faces, landscapes (e.g., Dag et al., 2010), or how it can perform fine motor skills activities (e.g., push, lift, grasps objects) (e.g., Detry et al., 2011). Equally important for the robot to function well in the environment is the ability to avoid certain objects, which has been termed the traversability affordance (Uğur \& Şahin, 2010). In other words, because sensing, planning, and executing are three major processes that robots must carry out to implement proper short-term responses and execute tasks in their environment (Brooks, 1986), the field of robotics has 
conceptualized affordances mostly in terms of these functions. Although recent approaches have considered how robots should deal with humans in the environment, however, this work is nonetheless still focused on physical domains (i.e., how a robot can use wheels to bypass or transit near a human, Lindler \& Eschenback, 2011).

In my view, though valuable, these approaches leave aside a more comprehensive perspective which considers the robots' capacity to act socially toward other social actors in the environment. Robots have recently undergone significant developments and have acquired the capacity to socially interact with humans and provide meaningful behaviors and responses (Guzman, 2018; Zhao, 2006). In line with previous scholars who have proposed to focus on the processes and effects emerging from the interaction between humans and robots (e.g., Edwards et al., 2019), I argue here that these new capabilities bring a new social affordance to social robots, that is their capacity to sustain meaningful social interactions with humans. To make this point, I bring together affordance approaches from computer-mediated communication (CMC) and human-robot interaction (HRI), to exemplify how these approaches see technology as a medium or tool through which humans can communicate.

\section{Bringing Approaches Together}

As I have shown, the concept of affordances has faced inconsistent focus in two importantly related fields relevant to HMC, such as robotics and communication. While one is objectbased, the other has been fully user-based. Further, even though we see enormous value in the relational approach to affordances such as those of Evans and colleagues (2016), I have shown how this approach could be made more specific in three important ways. Therefore, because this study's goal is to provide a more specific definition of the process of affordance formation, as it follows from the introduction to formally ask:

R.Q.1 = what is the process of enacted affordance formation applied to human-machine communication (HMC)?

As establishing conceptual clarity of the process of enacted communicative affordances represents a first step toward conceptualizing and identifying characteristics of social robots which may importantly influence affordances, we can deal now with the second goal of this theoretical article, which is to identify some crucial characteristics of social robots which may bring forth affordances. A characteristic which has been considered to be crucial in the definition of a social robot is that of physical embodiment. "Social robots are embodied agents ... able to recognize each other and engage in social interactions" (Fong et al., 2003, p. 144). This body enables them to both perceive and act socially in their environment (Paauwe et al., 2015). Further, an agents' corporality is intrinsically related to how the body allows the robot not only to sense its environment and act in response to it, but also to exert an action as an agent in the environment (Wiltshire et al., 2013).

In addition, physical embodiment appears to be closely tied to a sense of agency, which is linked to sensorimotor processes such as touch and movement. Touch and physical proximity allow a social robot to enter the persons' intimate physical sphere (Altman \& Taylor, 1973) and to be able to share interpersonal touch with humans. These haptic cues are 
essential to create a feeling of bonding, emotional warmth, and intimacy (Knapp \& Hall, 1992). These arguments may evidence that the social affordances of HMC would follow a different process compared to, for instance, communicating with a computer. Further, several studies have found that physically embodied robots appear to be more engaging and compelling to communicate with compared to an avatar, for instance (e.g., Kiesler et al., 2008). Following these considerations, this study's second research question is formally asked, following from the introduction:

R.Q.2 = what is the role of the embodiment in the process of enacted social robots' affordances?

\section{First Research Question: The Process of Enacted Communicative Affordance}

This article's first research question asked about the process of affordance formation, applied to human-machine communication, that is the human communicating with a social robot and vice versa. I consider this a two-way process and therefore the social robot is considered as an interaction partner with equal social standing than the human. Building on the affordance conceptualization by Faraj \& Azad (2012) and Evans et al. (2016), I provide here a definition of communicational affordances as both perceived and enacted possibilities for social interaction in a two-way iterative communication process, which emerges in the enactment of an integrated, sequential relational system which brings attitudinal, cognitive, and behavioral effects in both communication partners. Relevantly, because this is a communicational model in the context of HMC, I consider social affordances as emerging from a process of perceived and enacted communicative behaviors or actions between both partners, either the human or the social robot, which they perform in sequential fashion following an interaction and enabled by both their perception and their agency. In the case of the social robot, social agency is achieved through choosing from a myriad of interaction possibilities to respond or to initiate interaction with a human. Already, when facing communication with a human, social robots must choose between several alternatives which have been programmed. In the future, as social robots become more autonomous and sophisticated, their sense of all these interrelated steps will have increased importance and may become smoother and more automatized.

This paper definition of affordance considers communicational affordances as socially "enacted" because in my view, it does not suffice to just perceive possibilities for action with an object, it is actually the social actor (the person or the robot), who should ultimately enact the behavior to obtain a result from this social action. For instance, it does not suffice to perceive the social robot as a possible interaction partner, nor our intention and desire to talk to it which brings forth social affordances of meaningful social interaction. It is the combination of these factors plus their enactment which brings forth the social affordance of interactivity, for instance. For further clarity, I posit that the process outlined in Figure 1 would bring forth, "enact" a social affordance in the context of HMC.

Following the theoretical considerations presented in this paper, I first provide an account of the steps in the process of social affordance formation: (a) what the social agent 
perceives in the environment (the characteristics and features of the subject); (b) what that subject represents to the agent in terms of action possibilities, or the actions that the social agent perceives or imagines it can perform with the subject; (c) the actions that the social agent is willing to carry out (agency); (d) carrying out the interaction. After performing the social action, subsequently, the response of the second agent goes through the same sequence of steps (e, $f, g, h)$. If the social agent decides to respond $(h)$, then comes the result of the social action for the first agent (i).

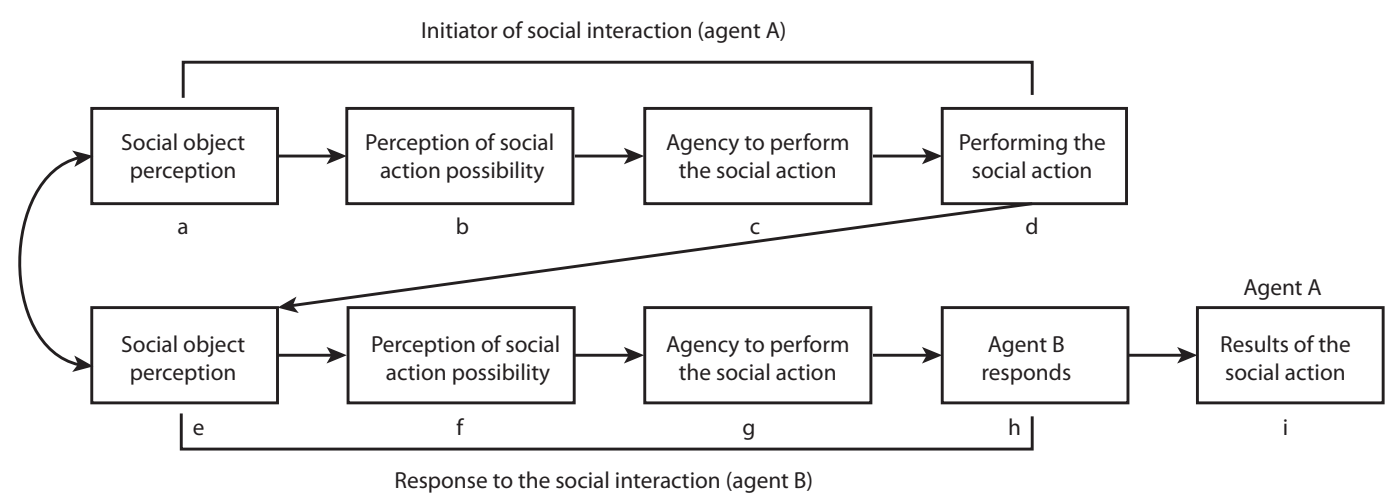

FIGURE 1 Model of Enacted Social Communicative Affordance Between a Human and a Social Robot and Vice Versa

The model of the process of enacted social affordance between a human and a social robot and vice versa, is seen on Figure 1. The semi-circular double-sided arrows to the left signal that this process is iterative, that is to say, after a social action is started by one social agent A (either the human or the social robot), the other agent B can choose to respond to the interaction and so on subsequently. First, the process takes place from the upper left square to the right. Then, as was already stated, after the social action by agent $\mathrm{A}$ is performed, the social agent B perceives this enacted social action and goes through the same process, from the down left square toward the right. All this process continues to the right until agent $B$ responds. Ultimately, this response from agent $\mathrm{B}$ ends up by having an outcome on agent A. We exemplify the model in more detail within the next research question. I approach research question 2, regarding the role of embodiment in the process of enacted affordance.

\section{Second Research Question: The Role of Embodiment in Social Affordances}

This study's second research question asked about the role of the embodiment in the process of enacted social robots' affordance. Importantly, this paper views stimulus or robot characteristics not as affordances. Rather, these characteristics represent stimuli which influence the process of communicative enacted affordances by making some aspects more salient (e.g., presence of a physical body, presence of a mouth). Although providing a complete 
account of the role of embodiment is out of scope in the present theoretical study, it is used here to exemplify the model of enacted social communicative affordance below.

a) What the social agent perceives in the environment (e.g., the characteristics and features of the subject).

Both the person and the social robot possess a body and social attributes such as body shape (e.g., presence of arms or legs), height, and facial features such as eyes and mouth. These elements will imply that both agents simply notice each other in a shared physical space.

b) What the social object represents to the agent in terms of action possibilities.

The physical characteristics of both agents makes it that both see each other as possible social interaction partners. Considering that agent $\mathrm{A}$ sees agent $\mathrm{B}$ as a social agent and vice versa, this implies a number of perceived or imagined interaction possibilities; for instance, physically approaching the subject, waving, making eye contact, or initiating a voice-based conversation.

c) The actions that the social agent is willing to carry out (agency).

After noticing distinct action possibilities and considering response possibilities of the other agent $B$, both agents will use their sense of agency to either begin an interaction or to respond to it. We posit that this agency will respond to varied personal goals of both agents, which range from socializing or learning new information. Embodiment plays a role here, because it enables action possibilities which include physicality; for instance, giving each other a hand, patting on the shoulder, or getting more proximally close.

d) Carrying out the social action.

Agent A initiates the social action, which for example can be making eye contact, touching agent $\mathrm{B}$, or initiating a dialogue. Embodiment enables these possibilities for social action because it crucially allows for embodied nonverbal language which can be encoded and decoded by both interaction partners. Crucially, it allows for physical proximity and touch of both communication partners. The robot is able to use its body to interact or send interaction signals. For instance, in the case of interacting with an anthropomorphic robot the agent has a number of "symbolic" features such as eyes or mouth to imply a certain nonverbal expression (i.e., interest), or can reach its arms to touch the person, or its legs to walk toward the person.

e) The results of this social action.

Lastly, the social action would bring a certain outcome. Agent B will respond to the social initiation by agent $\mathrm{A}$ and go through the same process as agent $\mathrm{A}$ to initiate a response. This response will trigger an outcome in agent A. Importantly, outcomes are conceived here as the behavioral, attitudinal/emotional, and cognitive effects 
emerging from the social interaction. Embodiment plays a key role in the behavioral component I argue, because if for instance the conversation is running smoothly and agent $\mathrm{A}$ receives a suitable response from agent $\mathrm{B}$, a behavioral reaction of agent $\mathrm{A}$ could be to decrease body distance between both agents, since they may increase their liking of the social robot. The emotional component is also affected by embodiment, as both parties presumably have a face and body, which can be used to express and read emotions by means of facial expressions in the other. Both agents' facial expressions and verbal communication have the potential of creating emotional outcomes in both partners. Lastly, cognitive effects are possible, provided that both agents, for example, cognitively process the interaction with one another, and for instance agent A, the human, learned new information thanks to the answer from agent B. An agent B can "read" and interpret both the verbal and nonverbal language provided by agent $\mathrm{A}$ and thus adapt their interaction depending on its goal.

\section{Discussion}

This theoretical paper had as its main aim to describe and define social communicative affordances with a focus on their formative process, in the setting of a social interaction between a human and a social robot. Further, it meant to identify a series of steps which may present themselves in the process of enacted communicative affordances in HMC. Although the present approach clearly has limitations-an important one being that its conceptualization is purely theoretical-I believe that a strength of our approach is that of presenting a comprehensive yet detailed account of the process of enacted communicative affordance formation in the context of HMC, considering the contributions of several affordance approaches. I am convinced that this approach can illuminate and enrich the discourse and research on HMC, for instance by distinguishing between perceived and enacted affordance and by presenting a scheme of the overall process of communicative affordance.

An innovative aspect of the present approach is that it considers the robot in equal social standing than the human in a communicative situation. Although this idea may seem unrealistic to some nowadays considering current social robot capabilities, particularly regarding their agency and nonverbal language expression, we believe that as social robots will become even more autonomous and social in the future, that the presented approach can be applied to better understand the new social affordances brought by social robots and can be of significant value to conceptualize perceived communicative affordances and assess effects from enacted affordances of HMC in the future.

The contributions of this study can be better understood by highlighting three conceptual remarks. These are: (1) stimulus or robot characteristics are not affordances; (2) robot characteristics influence possible enacted affordances; (3) the process of enacted affordances influence communication or interaction with robots and HMC outcomes. With regards to (1), it is not our aim to say that embodiment is an affordance per se, but rather that it is a salient social robot stimulus characteristic which is separate from perceived and enacted affordance. Although I am aware that the notion of embodiment may be rather controversial as accounting for social effects in robotics, it has been suggested that the very notion 
of an intelligent autonomous machine cannot exist without a body. "Intelligence requires a physical instantiation a body" (Pfeifer \& Scheier, 1999). Having a body would thus integrate the conceptualizations of possessing a social intelligence, and entails occupying the same physical 3-D space with the interaction partner and further allows for visual, auditory, and especially haptic nonverbal communication, all key aspects to make social robots seem more personable and able to establish meaningful social relationships with humans.

With regards to (2), as I have postulated, this paper proposes that social robots possess unique characteristics which may influence both perceived and enacted affordances. Examples have been provided regarding embodiment, but it is my intention to leave open for further research to identify which other unique social robot characteristics render which social affordances could be enacted through HMC. As for (3), and crucial to this study's proposition, affordances influence interactions with social robots and HMC outcomes. For instance, there is ground to assume that the affordances of interactivity and customization may bring a number of behavioral outcomes, such as more frequent interaction with the social robot, emotional outcomes such as greater closeness and liking, and/or lastly, cognitive outcomes such as learning.

Future work could test and/or expand this approach by attempting to establish the empirical relationship between perceived and enacted affordances in the context of HMC. Although this paper does not propose per se that affordances are measurable (a view toward which other scholars seem skeptical, considering the concept's relational structure), this paper poses that considering the steps of the affordance formation process (e.g., making the distinction between perceived and enacted affordance and the formation steps throughout), may be meaningful and at least theoretically relevant. In a similar fashion, future scholarship could consider whether and to what extent affordances commonly discussed in the context of other media (e.g., the locatability and portability of cell phones) (Schrock, 2015), play a role in the communicative affordance formation process of social robots. Lastly, to increase our knowledge in the field, this social affordance process could be discussed and researched in the context of communicating with other nonhuman forms of communicative agents, such as algorithms or virtual AI assistants. A last and important issue to consider is the context and factors in which enacted communicative affordances and their possible effects can be explicated. Obviously, situational, environmental, individual factors, and predispositions can affect enacted affordances. Ultimately, I am hopeful that by presenting a focused yet relational perspective to enacted affordances, this may help with comprehending how people interact and what they obtain from social robots, namely the capacity of meaningful social interaction. Important here is the notion that both interaction partners ascribe meaning to socially communicating with one another (Guzman, 2018). This is important to generate behavioral, emotional, and cognitive outcomes from interacting with social robots, outcomes which are very relevant to study now and in the future.

\section{Acknowledgments}

The author would like to thank Dr. Rinaldo Kühne from the Amsterdam School of Communication Research (ASCoR), University of Amsterdam, for his insightful comments throughout various stages of manuscript development. The author declares to have no conflict of interest. 


\section{Author Biography}

Carmina Rodríguez-Hidalgo, $\mathrm{PhD}, \mathrm{MSc}$, obtained her doctorate and research master degree in Communication Science at the University of Amsterdam, The Netherlands. Her research focuses on the emotional outcomes of mediated communication in youth and children. She recently joined Universidad Adolfo Ibáñez as an assistant professor in her native Chile to continue research on human-machine communication.

https://orcid.org/0000-0002-1690-9572

\section{References}

Altman, I., \& Taylor, D. A. (1973). Social penetration: The development of interpersonal relationships. Holt, Rinehart \& Winston.

boyd, D. (2010). Social network sites as networked publics: Affordances, dynamics, and implications. In A networked self (pp. 47-66). Routledge.

Brandi, M. L., Kaifel, D., Bolis, D., \& Schilbach, L. (2019). The interactive self-A review on simulating social interactions to understand the mechanisms of social agency. $i$-com, 18(1), 17-31. https://doi.org/10.1515/icom-2018-0018

Brooks, R. (1986). A robust layered control system for a mobile robot. IEEE journal on robotics and automation, 2(1), 14-23.

Cross, E. S., Hortensius, R., \& Wykowska, A. (2019). From social brains to social robots: Applying neurocognitive insights to human-robot interaction. Phil. Trans. R. Soc. B 374: 20180024. https://doi.org/10.1098/rstb.2018.0024

Dag, N., Atil, I., Kalkan, S., \& Sahin, E. (2010, August). Learning affordances for categorizing objects and their properties. In 2010 20th International Conference on Pattern Recognition (pp. 3089-3092). IEEE.

Detry, R., Kraft, D., Kroemer, O., Bodenhagen, L., Peters, J., Krüger, N., \& Piater, J. (2011). Learning grasp affordance densities. Paladyn, Journal of Behavioral Robotics, 2(1), 1-17. https://doi.org/10.2478/s13230-011-0012-x

Edwards, A., Edwards, C., Westerman, D., \& Spence, P. R. (2019). Initial expectations, interactions, and beyond with social robots. Computers in Human Behavior, 90, 308-314. https://doi.org/10.1016/j.chb.2018.08.042

Evans, S. K., Pearce, K. E., Vitak, J., \& Treem, J. W. (2016). Explicating affordances: A conceptual framework for understanding affordances in communication research. Journal of Computer-Mediated Communication, 22(1), 35-52. https://doi.org/10.1111/jcc4.12180

Faraj, S., \& Azad, B. (2012). The materiality of technology: An affordance perspective. In Leonardi, P., Nardi, B., and Kallinikos, J. (Eds.), Materiality and organizing: Social interaction in a technological world, Oxford University Press, pp. 237-258.

Fodor, J. A., \& Pylyshyn, Z. W. (1981). How direct is visual perception? Some reflections on Gibson's "ecological approach." Cognition, 9(2), 139-196.

Fong, T., Nourbakhsh, I., \& Dautenhahn, K. (2003). A survey of socially interactive robots. Robotics and autonomous systems, 42(3-4), 143-166.

Fox, J., \& Holt, L. F. (2018). Fear of isolation and perceived affordances: The spiral of silence on social networking sites regarding police discrimination. Mass Communication and Society, 21(5), 533-554. https://doi.org/10.1080/15205436.2018.1442480 
Gibson, J. J. (1986). The ecological approach to the visual perception of pictures. Original work published 1979. New Jersey: Lawrence Erlbaum Associates.

Guzman, A. L. (2018). What is human-machine communication, anyway? In A. L. Guzman (Ed.), Human-machine communication: Rethinking communication, technology, and ourselves (pp. 1-28). Peter Lang.

Horton, T. E., Chakraborty, A., \& Amant, R. S. (2012). Affordances for robots: A brief survey. AVANT. Pismo Awangardy Filozoficzno-Naukowej, 2, 70-84.

Kiesler, S., Powers, A., Fussell, S. R., \& Torrey, C. (2008). Anthropomorphic interactions with a robot and robot-like agent. Social Cognition, 26(2), 169-181. https://doi.org/10.1521/ soco.2008.26.2.169

Knapp, M. L., \& Hall, J. A. (1992). Nonverbal Communication in Human Interaction. Wadsworth.

Kuindersma, S., Deits, R., Fallon, M., Valenzuela, A., Dai, H., Permenter, F., Koolen, T., Marion, P., \& Tedrake, R. (2016). Optimization-based locomotion planning, estimation, and control design for the atlas humanoid robot. Autonomous Robots, 40(3), 429455. https://doi.org/10.1007/s10514-015-9479-3

Lindner, F., \& Eschenbach, C. (2011, September). Towards a formalization of social spaces for socially aware robots. In International Conference on Spatial Information Theory (pp. 283-303). Springer, Berlin, Heidelberg.

Nagy, P., \& Neff, G. (2015). Imagined affordance: Reconstructing a keyword for communication theory. Social Media + Society, 1(2), https://doi.org/10.1177/2056305115603385

Norman, D. A. (1999). Affordance, conventions, and design. interactions, 6(3), 38-43.

Norman, D. A. (November 17, 2008). Affordance, conventions and design. Archived at https://web.archive.org/web/20190119062638/https://jnd.org/affordance_conventions_ and_design_part_2/

Paauwe, R. A., Hoorn, J. F., Konijn, E. A., \& Keyson, D. V. (2015). Designing robot embodiments for social interaction: Affordances topple realism and aesthetics. International Journal of Social Robotics, 7(5), 697-708. https://doi.org/10.1007/s12369-015-0301-3

Peter, J., \& Kühne, R. (2018). The new frontier in communication research: Why we should study social robots. Media and Communication, 6(3), 73-76. https://doi.org/10.17645/ mac.v6i3.1596

Pfeifer, R., \& Scheier, C. (1999). Understanding intelligence. MIT Press.

Pols, A. J. (2012). Characterising affordances: The descriptions-of-affordances-model. Design Studies, 33(2), 113-125. https://doi.org/10.1016/j.destud.2011.07.007

Schrock, A. R. (2015). Communicative affordances of mobile media: Portability, availability, locatability, and multimediality. International Journal of Communication, 9, 1229-1246.

Smock, A. D., Ellison, N. B., Lampe, C., \& Wohn, D. Y. (2011). Facebook as a toolkit: A uses and gratification approach to unbundling feature use. Computers in Human Behavior, 27(6), 2322-2329. https://doi.org/10.1016/j.chb.2011.07.011

Stoffregen, T. A. (2003). Affordances as properties of the animal-environment system. Ecological psychology, 15(2), 115-134. https://doi.org/10.1207/S15326969ECO1502_2

Uğur, E., \& Şahin, E. (2010). Traversability: A case study for learning and perceiving affordances in robots. Adaptive Behavior, 18(3-4), 258-284. https://doi. org/10.1177/2F1059712310370625 
Vyas, D., Chisalita, C. M., \& Van Der Veer, G. C. (2006, September). Affordance in interaction. In Proceedings of the 13th European conference on Cognitive ergonomics: Trust and control in complex socio-technical systems (pp. 92-99). ACM.

Wiltshire, T. J., Barber, D., \& Fiore, S. M. (2013, September). Towards modeling socialcognitive mechanisms in robots to facilitate human-robot teaming. In Proceedings of the human factors and ergonomics society annual meeting (Vol. 57, No. 1, pp. 12781282). SAGE Publications.

Zhao, S. (2006). Humanoid social robots as a medium of communication. New Media e Society, 8(3), 401-419. https://doi.org/10.1177/1461444806061951 

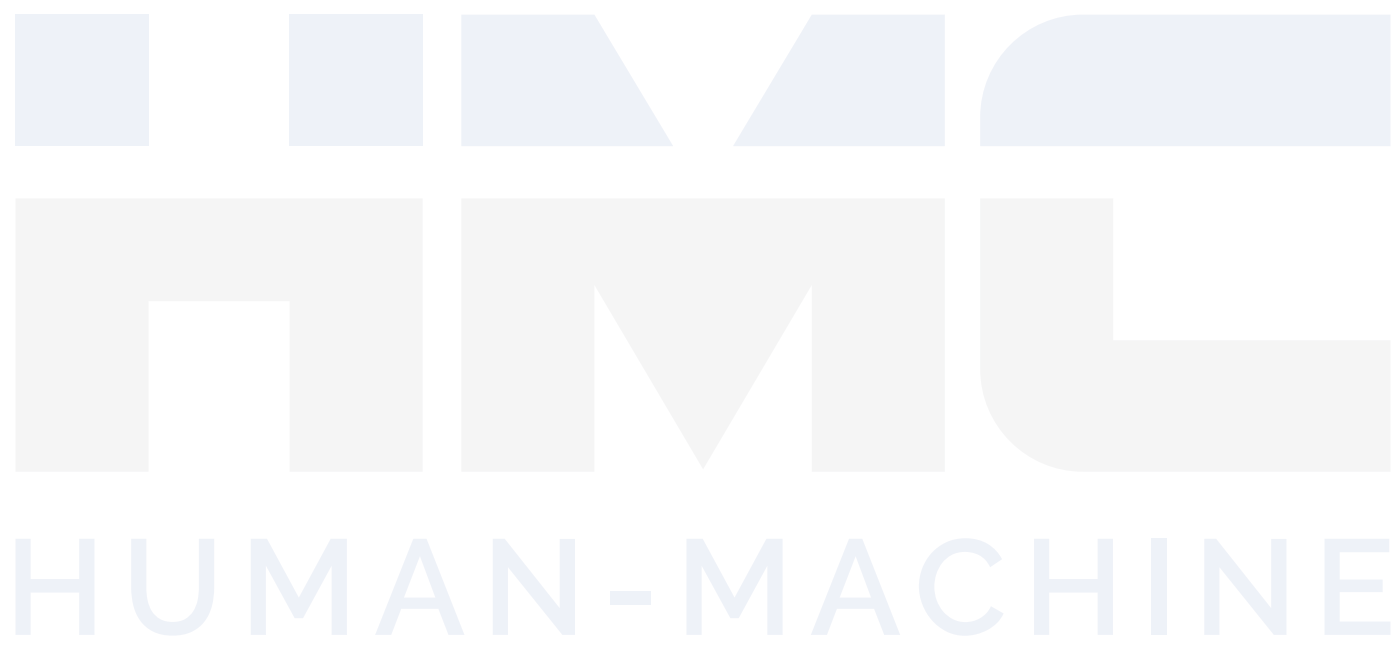
COMMUNHCATHON 